\title{
DECISION MAKING ON DISASTER MANAGEMENT IN AGRICULTURE WITH SENTINEL APPLICATIONS
}

\author{
G. Doxani ${ }^{1, *}$, S. Siachalou ${ }^{1}$, Z. Mitraka ${ }^{2}$, P. Patias ${ }^{1}$ \\ ${ }^{1}$ Department of Rural \& Surveying Engineering, Aristotle University of Thessaloniki, Thessaloniki, Greece - (gdoxani, ssiacha, \\ patias)@auth.gr \\ ${ }^{2}$ Foundation for Research and Technology, Hellas, Institute of Applied and Computational Mathematics, N. Plastira 100, Vassilika \\ Vouton, P.O. Box 1385, GR-71110, Heraklion, Greece- mitraka@iacm.forth.gr
}

Commission VI, WG VI/4

KEY WORDS: Disaster Monitoring, Agriculture, Sentinels, Decision Making, Compensations

\begin{abstract}
:
Climate change and increase of extreme weather events, besides the numerous consequences, affect significantly and put in risk the agriculture sectors. Natural disasters, such as floods and wildfires, are responsible for a great loss in agriculture production. National governments together with international bodies make an important effort to cooperate towards the response and resilience when a disaster occurs. In this frame the European Earth Observation Programme - Copernicus provides a series of observation data, in-situ measurements and services related, amongst others, to different types of disasters. Concerning the availability of this big volume of observation data, the aim of DiAS (Disaster and Agriculture Sentinel Applications) project is to revise the existing knowledge on remote sensing methods for mapping the extent of natural and/or man-made disaster over agricultural areas and propose improvements. The developed methodology will be implemented in a Decision Support System (DSS), which will be freely available and easy-to-use by non-experts. In this paper, the developed methodology focuses on mapping floods over agricultural areas. Sentinel-1 and Sentinel2 imagery are used as input information for the comparison analysis before and after the event. The reference for results' evaluation is the corresponding information delivered by Copernicus Emergency Management Service (EMS). Although, the evaluation results are in good agreement when they could be used, a reference of higher accuracy is needed in order to estimate accurately the quality of the output products.
\end{abstract}

\section{INTRODUCTION}

Considering the high importance of the agricultural sector, the European Union (EU) implements a set of regulations under the Common Agricultural Policy (CAP), aiming at the sustainable development and management of natural resources. In the case of a natural disaster, EU and each state member offer recovery assistance to disaster-affected farmers to mitigate the impact of the problems caused. The principles of CAP and the implementation of compensation measures require precise detecting and monitoring of natural disasters' effects by carrying out timely and accurate controls. Until now, in Greece for instance, these controls are mainly performed by in-situ visits resulting in very high administrative costs. This procedure is not only time-consuming itself, but certain difficulties, i.e. adverse weather conditions, inaccessible areas, etc., can further delay the compensations. Copernicus Emergency Management Service provides operationally today mapping information for disasteraffected areas upon request of authorised users at regional, national, European and international level. However, the service is activated under certain circumstances, i.e. extent, damages, duration, etc. and does not cover all the disaster events.

Free and open access policy to Copernicus Sentinel data offers a big volume of data to the users on a consistent and complete basis. Due to their various features and the frequent revisit time, Sentinels support efficiently many environmental applications, including monitoring of agricultural fields and mapping floods and fire extent.

Concerning flood events, the synthetic aperture radar (SAR) data are the key information input for the mapping the extent of the flood, as cloud coverage hinders the usage of optical data in these cases. There are various approaches developed for the automatic flood detection (Hess et al. 1995, Martinis et al. 2015, Benoudjit et al., 2019) and many emerged after the free and open access to Copernicus Sentinel-1A and -1B data. A major advantage of the constellation is the systematic data acquisition every 6 days. In addition, the data is available within an hour of reception for Near Real-Time (NRT) emergency response, within three hours for NRT priority areas and within 24 hours for systematically archived data. This means that the time interval between occurrence of the disaster event and coverage can be significantly reduced.

Based on Sentinel-1 data, Twele et al. (2016) developed an automated methodology towards the flood detection and mapping. After applying all the necessary steps of geometric and radiometric calibration, an automatic thresholding classified the image to 'water' and 'no water' areas, which were later refined thanks to a fuzzy-logic-based classification and auxiliary data. The performance of the proposed algorithm was evaluated with the help of a manually produced water extent map derived from WorldView-2 data. In the comparison analysis, differences between $\mathrm{VV}$ and $\mathrm{VH}$ polarization were observed, with the classification results based on VV-polarized data to reach higher user's, producer's, and overall accuracies.

Amitrano et al. (2018) proposed an approach based on two processing levels of Sentinel-1 images providing flood maps with increasing resolution. At the first level a fuzzy classification system handles the co-occurrence texture measures and amplitude information for defining 'Flood' and 'No Flood' areas. At the second processing level a change index between the preand post-event images is calculated and added to the fuzzy classification system. In both levels an object-based image 
analysis (OBIA) is applied on the final map to eliminate the small areas according to a user-defined threshold. The implementation results were compared with Copernicus Emergency Management Service (EMS) data and other methods, i.e. supervised support vector machine (SVM), neural net $(\mathrm{NN})$, maximum likelihood (ML) and unsupervised k-mean, with very high accuracy results. Taking advantage of the valuable information in Sentinel-1 time series, Tsyganskaya et al. (2018) developed an approach based on pixel- and object-image analysis for the detection of flood classes: Temporary Flooded Vegetation and Temporary Open Water. Pixel-based analysis included the calculation of the mean value, median, and standard deviation of backscatter values over the time series. These estimations were the input to a Random Forest classification towards identifying the classes of interest. In parallel, the object-image analysis was running by implementing a clustering approach on the combination of the VV and VH time series layer stacks. A further thresholding process and auxiliary data refined the classification results. The performance analysis was conducted by comparing the classification results to a Sentinel-2 reference flood map achieving promising results.

DiAS research project aims to develop a processing chain for mapping the agricultural crops and the extent of natural and/or man-made disasters over agricultural areas. The classification tool is designed for users that are not remote sensing experts, like the decision makers on farmers' subsides and compensations in the corresponding Greek services, e.g. the Greek Agricultural Insurance Organization. In this paper we will focus on the methodology developed to a detailed mapping product showing the extent of floods and their effects in agricultural land. The goal in this case is to develop a tool for the authorized agencies to support field visits by providing additional evidences, when a disaster occurs. Today these visits are realized following the farmers' indications and descriptions, a tactic that requires time and man power. A field visit is usually important for the crop assessment and compensation approval in these cases, in order to estimate the effects on crop yields as a flood may not necessary destroy the productivity of the entire field. Therefore, in our case, it is more important to detect the location of the flood rather than the exact extent area. In particular, the flooded areas are mapped using Sentinel-1 and Sentinel-2 data, following a comparison procedure of the images acquired pre- and post- flood event. Filtering and thresholding are implemented on the Sentinel-1 difference image of the two dates, with the aim to highlight the areas covered with water. In order to improve this detection, a water index is calculated based on the corresponding Sentinel-2 data providing this way ancillary information for more accurate mapping.

\section{METHODOLOGY}

The aim of the project is to develop a tool to be easily used by non-remote sensing experts, so it can be automatically implemented in Python calling SNAP code and QGIS. The workflow of the proposed processing chain, as outlined in Figure 1.

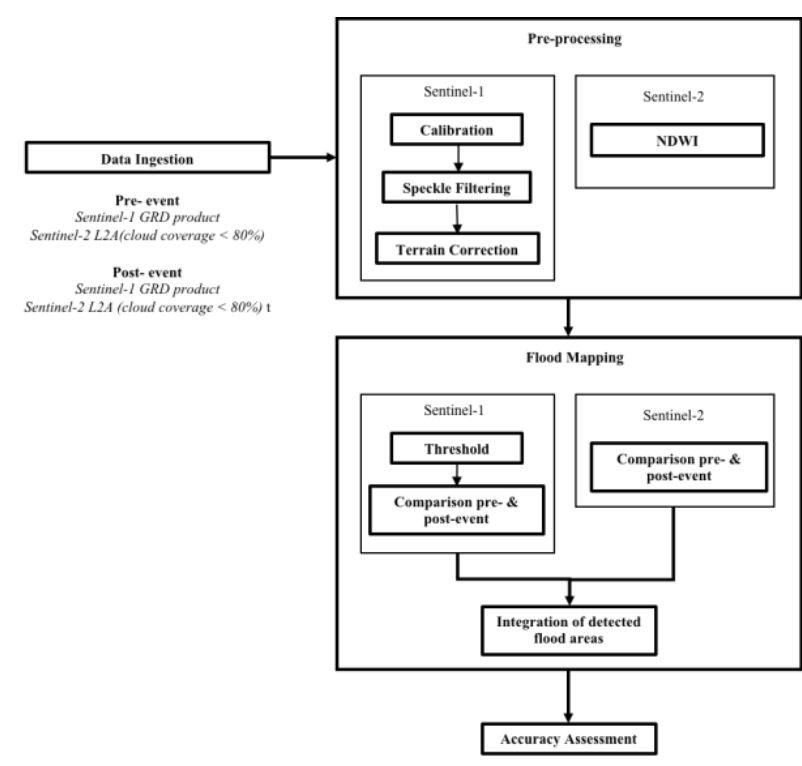

Figure 1. Flowchart of the proposed flood mapping processing chain

\subsection{Input data}

Copernicus Sentinel data provided the input information in the proposed processing chain. In particular, Sentinel-1 Level-1 Ground Range Detected (GRD) products were mainly used to analyse the flood events, due to their availability in any weather conditions. When the cloud coverage permitted, atmospherically corrected Sentinel-2 L2A products contributed to detect and map flood extent. An algorithm checks and downloads automatically from Copernicus Open Access Hub the available products before and after the event. User intervention is limited to defining the area and product of interest by setting the proper parameters, i.e. coordinates, dates, product type, etc

\subsection{Data pre-processing}

Radiometric calibration was implemented to Sentinel-1 images, in order to obtain pixel values with physical meaning, i.e. backscatter values. This step is necessary when we need to extract quantitative information from radar images and be able to analyse time series. Speckle noise reduction was then applied by a 5 x 5 Lee spatial filter. This way the salt-and-pepper effect reduced on the image, improving its quality and the interpretation of the scene features. A histogram conversion from a linear to a $\log$ distribution (DN to $\mathrm{dB}$ ) facilitated also the extraction of image information, as the distribution of the data is less skewed than in the linear scale. Terrain correction was the final preprocessing step to eliminate the image distortions and project the image pixels to the corresponding map projection.

In the cases of image cloud coverage less than 80 per cent before and after the event, Sentinel-2 atmospherically corrected products (L2A) were directly requested via Copernicus Open Access Hub. The calculation of Normalised Difference Water Index (NDWI) (Eq.1) as proposed by McFeeters (1996) highlighted the water areas.

$$
N D W I=\frac{\text { Green }-N I R}{\text { Green }+N I R}
$$

For Sentinel-2 bands, Eq.1 corresponds to:

$$
N D W I=\frac{\text { Band } 3-\text { Band } 8}{\text { Band } 3+\text { Bnad } 8}
$$




\subsection{Flood mapping}

A threshold was set on the pre-processed S-1 images based on their histogram, in order to detect the water areas on pre- and post-event images. Then based on the logical expression "if 'no water' on datel and 'water' on date 2 then 'flood'," the 'water' pixels were flagged as 'flood' on the post-event image when they were not corresponding to any type of water body on the preevent image.

In the resulted NDWI images water body areas were successfully pointed out with brighter pixel values. McFeeters (1996) developed the index based on Landsat MS image bands and suggested to consider as water the pixel values greater than zero. In case of Sentinel-2 images this threshold can lead to misleading information, classifying building rooftops and shadows, dark objects or asphalt, can be classified as water (Benoudjit, 2019; Kaplan, 2017). Taking into account these commission errors, the threshold for NDWI images was set to 0.2 , meaning that NDWI pixel values greater than 0.2 were assigned to 'water' class and the rest to 'other class'.

\subsection{Accuracy assessment}

The quantitative evaluation of the resulted flooded maps was performed with the help of the data delivered by Copernicus Emergency Management Service (EMS) - Mapping activations over Greece (https://emergency.copernicus.eu). The service provides free and open access information, including maps and vector files, shortly after a disaster occurs. The service should be activated by local delegates and then mapping information is extracted by utilising satellite imagery and other geospatial data.

There is a variety of statistical metrics in the literature to evaluate classification results with the most popular being Overall Accuracy and Kappa coefficient (Stehman, 1997) calculated based on the confusion matrix. In our case the reference corresponded to polygons of a vector file, so the evaluation was based on similarity metrics for objects matching (Batini, 2017; Cai et al., 2018). Therefore Completeness, Correctness and Quality metrics were calculated based on True Positives ( $\mathrm{TP}=$ the number of output pixels correctly assigned to the 'flood' class), False Negatives ( $\mathrm{FN}=$ the number of output pixels incorrectly assigned to the 'no-flood' class), False Positives areas (FP=the number of output pixels incorrectly assigned to the 'flood' class).

$$
\begin{aligned}
& \text { Completeness }=\frac{T P}{T P+F N} \\
& \text { Correctness }=\frac{T P}{T P+F P} \\
& \text { Quality }=\frac{T P}{T P+F N+F P}
\end{aligned}
$$

\subsection{Implementation}

The methodology of the DiAS project, both for the crops classification and flood mapping is implemented in a Decision Support System (DSS). The DiAS DSS aims at providing readily information to the respective authorised agencies. Thus it is expected to improve the efficiency of the decision-making process on agricultural aid and reimbursement by reducing the number of in-situ visits, minimizing costs and delays of controls. The pre-processing of the SAR and optical images is applied by calling SNAP operators via Python scripts. The DiAS DSS will be implemented as a QGIS plugin and will require minimum parametrization for the flood mapping. Therefore, the implementation of the entire processing chain will be free and open access. Guidelines will be provided to the user to decide on a) the imagery to use, b) the method parametrization to apply and c) the interpretation of the results.

\section{EXPERIMENTAL RESULTS}

\subsection{Study cases and datasets}

The following cases of Copernicus EMS- Mapping were considered in this study:

1. Chrisoupoli, North Greece (Activation Code: EMSR292) Heavy rainfalls in the area inundated thousand acres of agricultural areas and parts of the national road network. The event occurred on 28-06-2018 and the service was activated few hours later. The flood extent information was derived based on a Sentinel-2 image acquired on 25/06/2018 (pre-event) and a Sentinel-1B acquired on 29/06/2018 (post-event), both provided under Copernicus by the European Union and ESA (Figure 2).

2. Alfeios River, Western Greece (Activation Code: EMSR337)A flood was reported on 12-01-2109 due to an overflow of Alfeios river. Many agricultural fields along the riverbank were covered by water for many days after the initial event, according to the local authorities. The flood maps were produced based on a Sentinel-2 image acquired on 26/10/2018 (pre-event) and a PlanetScope ${ }^{\odot}$ Planet image acquired on 15/01/2019 (post event), both provided under Copernicus by the European Union and ESA.

\subsubsection{North Greece, Chrisoupoli case}

The flood event in this case was reported on 28-06-2018. The algorithm checked for available Sentinel- 1 data in dates \pm 6 days (S1 temporal resolution) from the event date and for Sentinel-2 in dates \pm 5 days ( 2 temporal resolution). An additional parameter for Sentinel-2 selection was the image cloud coverage to have been less than 80 per cent. The available Sentinel images are presented in Table 1. The study area was covered by two neighbouring Sentinel-2 granules, therefore both were downloaded and analysed.

\begin{tabular}{|c|c|c|c|c|}
\hline \multirow{2}{*}{} & \multicolumn{2}{|c|}{ Sentinel-1A, -1B } & \multicolumn{2}{c|}{ Sentinel-2A, -2B } \\
\cline { 2 - 5 } & Pre- & Post-event & Pre- & Post-event \\
\hline Date & $23-06-2018$ & $29-06-2018$ & $25-06-2018$ & $30-06-2018$ \\
\hline Product & GRD & GRD & MSI-L2A & MSI-L2A \\
\hline Orbit & 7 & 7 & 50 & 50 \\
\hline Granule & - & - & $\begin{array}{c}\text { T35TKF, } \\
\text { T35TLF }\end{array}$ & $\begin{array}{c}\text { T35TKF, } \\
\text { T35TLF }\end{array}$ \\
\hline $\begin{array}{c}\text { Cloud } \\
\text { Percent }\end{array}$ & - & - & $37.00,3.15$ & $27.03,34.9$ \\
\hline
\end{tabular}

Table 1. The features of the Sentinel input data for Chrisoupoli case

In Figure 2, the flooded areas, as estimated by Copernicus EMS, are highlighted with magenta colour and overlaid on the Sentinel1 image after the event (29-06-2018). According to the service the extent of the affected area was 1689.2 ha was and corresponded mainly to agricultural areas scattered in the valley of river Nestos. As the interest of this study concentrates on the detection of the inundated agricultural fields, the corresponding information of the Land Parcel Identification System (LPIS) was taken into consideration during the analysis. 


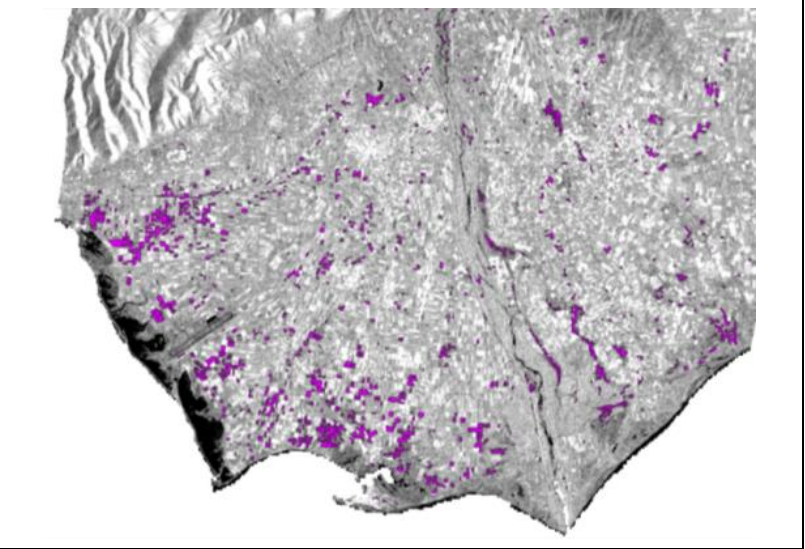

Figure 2. The available information of Copernicus Emergency Management Service (ㄷ 2015 European Union), [EMSR292] Chrisoupoli: Delineation Map (in magenta) on Sentinel-1 image (29-06-2018)

\subsubsection{Western Greece, Alfeios West case}

Due to high cloud coverage around the date of the event (12-012019), none Sentinel-2 image was available, so the analysis in this case was performed by using only Sentinel-1 images acquired on 06-01-2019 (pre-event) and 12-01-2019 (postevent). According to Copernicus EMS the time of event was at 08:00 (UTC) and Sentinel-1 image acquisition time was 16:30 (UTC), so we can consider it as a post-event even if it was obtained the same day.

The flooded areas as calculated by Copernicus EMS are shown in magenta colour and superimposed on the Sentinel-1 image (12-01-2019) (Figure 4). A total inundated area of 101.1 ha was reported by the service. It is worth mentioning that the activation of the service was on 14-01-2019 and the maps are referred to the situation as it was on 15-01-2019. LPIS information has been requested, but still not available for this study case.

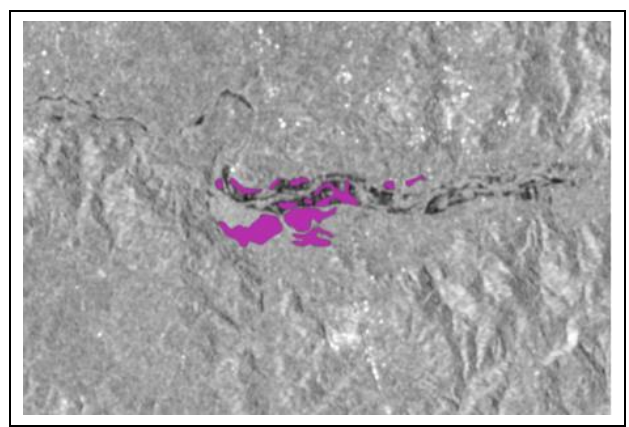

Figure 3. The available information of Copernicus Emergency Management Service (ㄷ 2015 European Union), [EMSR337] Alfeios: Delineation Map (in magenta) on Sentinel-1 image (1201-2019)

\subsection{Results}

\subsubsection{North Greece, Chrisoupoli case}

Initially, only two of the available images (Table 1) participated in the analysis, i.e. Sentinel-2 image before and Sentinel-1 image after the event, similarly to Copernicus EMS input data. The Sentinel-1 image was calibrated, speckle filtered and terrain corrected according to the processing chain described in $\S 2$. A threshold set to the output image and the water areas detected were refined based on the NDWI information of the pre-event Sentinel-2 image. This way the existing water bodies eliminated in the area of interest and only flooded areas were highlighted. A subset of the detected inundated areas is presented in Figure 4 corresponding to Copernicus EMS (in red) and the proposed approach using the same input data (in pink).

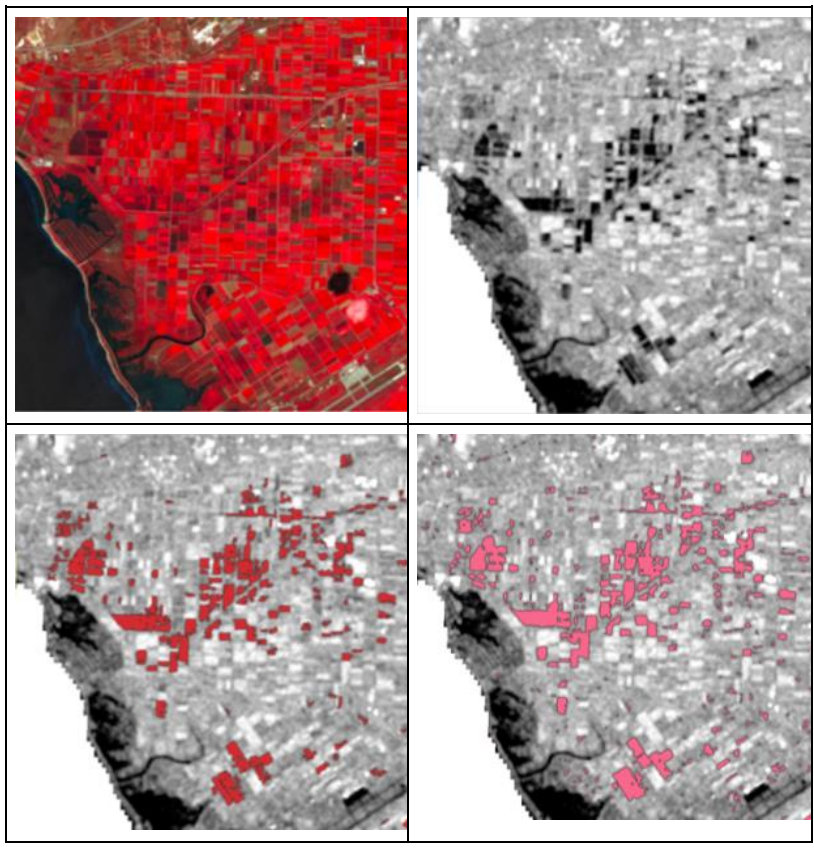

Figure 4. Sentinel-2 pre-event, pseudo-colour image visualisation (upper left), Sentinel-1 post-event processed image (upper right), Copernicus EMS vector output in red (lower left) and proposed methodology's vector output in pink (lower right) over a subset of the area of interest.

Both Copernicus EMS and our study's output were masked by the available outline of the agricultural fields (LPIS), so as to ignore the rest of land cover types and focus on the effect over agricultural fields. The results of the accuracy assessment analysis are presented in Table 2.

\begin{tabular}{|l|c|}
\hline Metrics & Value \\
\hline Completeness & 0.818 \\
\hline Correctness & 0.792 \\
\hline Quality & 0.674 \\
\hline
\end{tabular}

Table 2. The Completeness, Correctness and Quality metrics estimation for the inundated agricultural fields detected by the proposed methodology.

In Figure 5, the correctly detected flooded areas are presented in cyan colour, the incorrectly ones in red and the omitted areas in yellow. The proposed methodology succeeded in detecting most of the flooded areas. There is, though, an area of around 200 ha (Table 3) that was falsely classified as flooded. Most of the committed and omitted pixels were referred to parts of correctly detected flooded fields. Therefore it should be mentioned that the position of the flooded fields was detected correctly, but not the exact shape and area. 


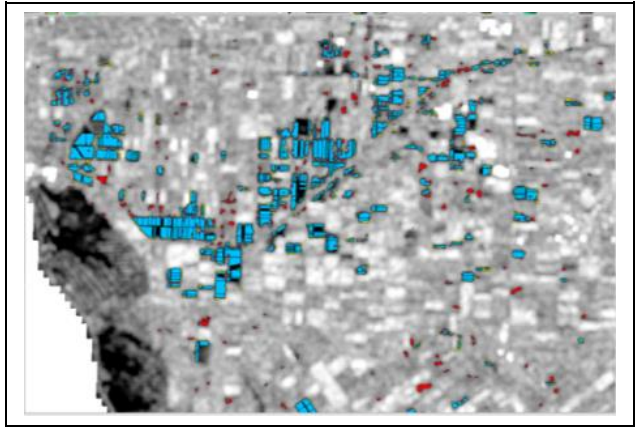

Figure 5. A closer view of an inundated agricultural area. TP pixels are presented with cyan colour, FP with red and FN with yellow colour.

\begin{tabular}{|l|c|c|}
\hline Product & No of fields & Flooded Agri Area \\
\hline Copernicus EMS & 3499 & 1006.7 ha \\
\hline Output Map & 4000 & 1222.41 ha \\
\hline
\end{tabular}

Table 3. The number and area of agricultural fields detected as flooded by Copernicus EMS and this study.

Besides SAR imagery, in the Chrisoupoli case-study, Sentinel-2 post event images were also available, but they were not used by Copernicus EMS. Figure 6 shows a visual comparison of our approach and the Copernicus EMS results. By superimposing the vector file of the flooded pixels assessed by Copernicus EMS in cyan on the Sentinel-2 image acquired two days after the event (30/06/2018) some omitted regions can be noted. Some of them are highlighted in the yellow. There areas were failed to be detected by analyzing only the SAR image, probably because of the presence of vegetation, trees or other elements that affected the signal. (Figure 6)
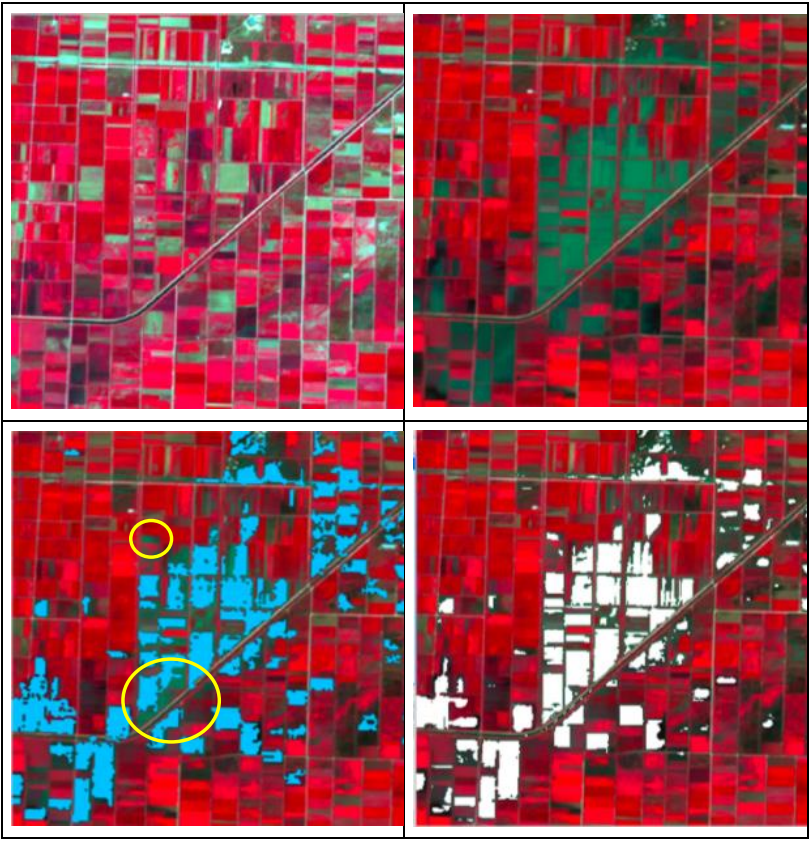

Figure 6. Sentinel-2 pre-event (upper left) and post-event (upper right) pseudo-colour visualizations, Copernicus-EMS vector output in cyan (lower right) and our approach's output in white (lower left) over a subset of the area of interest.
In the developed approach, all the available SAR and optical images participate in the detection of flooded areas. . The inundated regions which are shown in Figure 6 in white, resulted by following the processing steps described in $\$ 2$ and by incorporating all the data of Table 1 , However, the evaluation of the results need further investigation in this case. As Copernicus EMS cannot assure for the accuracy of the providing products, we intend to digitize the inundated areas based on Planetscope satellite imagery (spatial resolution: $3 \mathrm{~m}$, spectral resolution: RGB, NIR) and assess the accuracy of our products.

\subsubsection{Western Greece, Alfeios West case}

Following the SAR pre-processing steps described in in $\S 2$, the water areas were clustered in both pre- and post-event images. A comparison between them eliminated existing water pixels and flooded areas were highlighted (Figure 7).

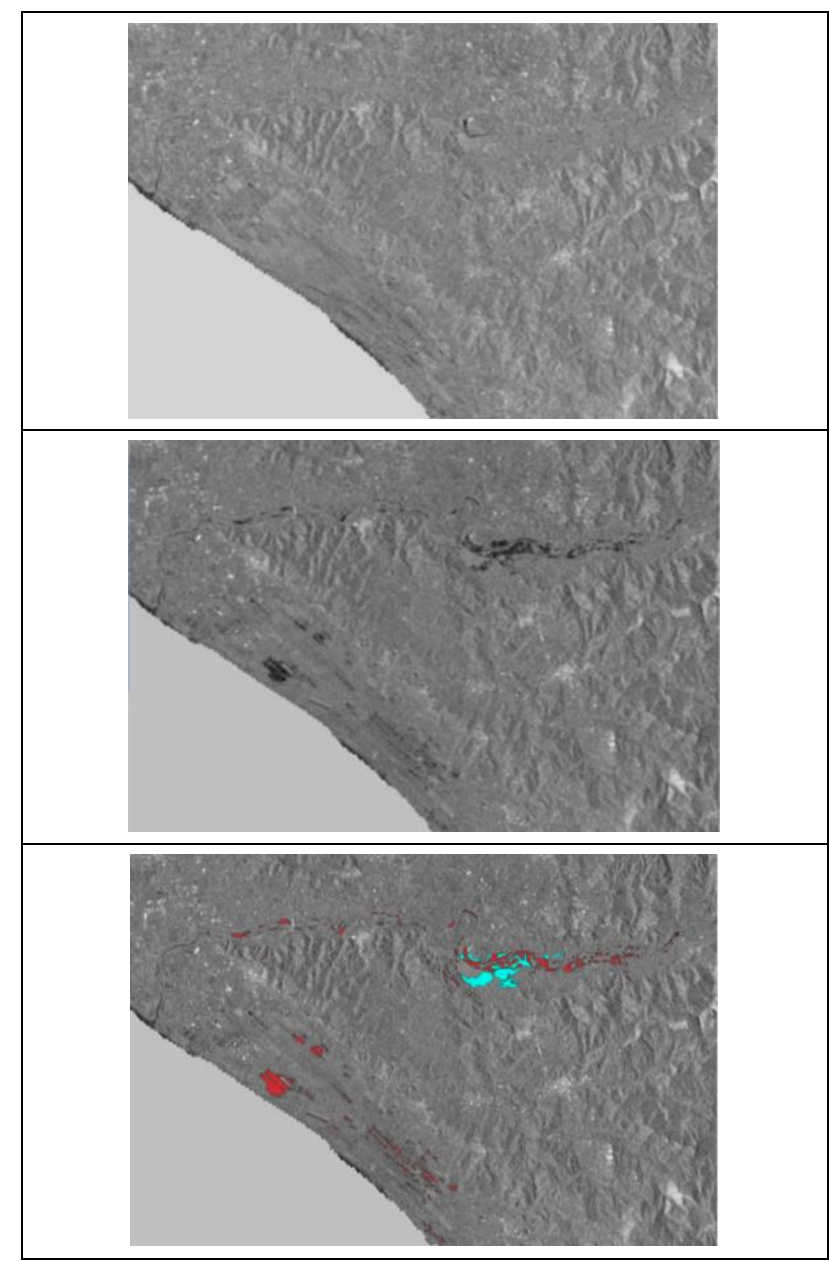

Figure 7. Sentinel-1 pre-event (upper), post-event (middle) and Copernicus-EMS vector output in cyan (lower) and our approach's output in red (lower) over the area of interest.

By these images' interpretation, it is evident that Copernicus service omitted parts of the flooded areas on 12-01-2019. This is probably because the service mapped the situation as it was on 15-01-2019, so some of the water could have retreated. Comparing the detected areas, the extent of the flood mapped by the proposed methodology is almost four times bigger than the one mapped by Copernicus-EMS. Regarding the differences on the dates studied and the input data between the output and the reference products, the accuracy assessment omitted in this case. 


\begin{tabular}{|l|c|}
\hline Product & Flooded Area \\
\hline Copernicus EMS & 101.1 ha \\
\hline Output Map & 432.445 ha \\
\hline
\end{tabular}

Table 4. The area of inundated areas detected by CopernicusEMS and this study's approach.

\section{CONCLUSIONS}

The proposed methodology aims to introduce a processing chain for the analysis of Sentinel data towards flood detection. The interest focuses on agricultural areas, so the developed tool will assist the decision makers on farmers' compensations. Up to date the controls for the inundated crops are made mainly by filed visits at the entire affected area and/or by using Copernicus-EMS product when available. The DSS under development in the current project will provide the location and extent of the area affected by a disaster, facilitating this way the task of the corresponding agencies.

In this paper, only two flood cases in Greece are analysed and compared to Copernicus-EMS output. The objective is to have investigated and evaluated all the eight flood events covered by the service in Greece since 2015. However, we figured out during these studies that Copernicus output is not always accurate and reliable to be used blindly as a reference. This deduction is confirmed by the service, which explicitly states that the usage of its products is upon users' responsibility and their accuracy is not guaranteed. Therefore, besides the service, high and very high resolution (when available) will be used in the future to digitise the inundated areas. A higher accuracy reference will assist in improving our approach and the reliability of our products.

\section{ACKNOWLEDGEMENTS}

This research is co-financed by Greece and the European Union (European Social Fund- ESF) through the Operational Program «Human Resources Development, Education and Lifelong Learning 2014-2020» in the context of the project "DIsaster \& Agriculture Sentinel applications- DIAS" (5004813). The authors would like to thank the Greek Payment Authority of Common Agricultural Policy Aid Schemes of the Ministry of Rural Development and Food for providing reference data from the Land Parcel Identification System (LPIS).

\section{REFERENCES}

Amitrano, D., Di Martino, G., Iodice, A., Riccio, D., Ruello, G., 2018: Unsupervised Rapid Flood Mapping Using Sentinel-1 GRD SAR Images. IEEE Transactions on Geoscience and Remote Sensing, 56, 6, 3290-3299. doi.org/10.1109/TGRS.2018.2797536.

Batini, C., Blaschke, T., Lang, S., Albrecht, F., Abdulmutalib, H. M., Barsi, Á., Szabó, G., Kugler, Z., 2017: Data quality in remote sensing. ISPRS - International Archives of the Photogrammetry, Remote Sensing and Spatial Information Sciences, XLII-2/W7, 447-453.

Benoudjit, A., Guida, R. A., 2019: Novel Fully Automated Mapping of the Flood Extent on SAR Images Using a Supervised Classifier. Remote Sens. 11, 779. doi.org/10.3390/rs11070779.
Cai, L., Shi, W., Miao, Z., Hao, M., 2018: Accuracy Assessment Measures for Object Extraction from Remote Sensing Images. Remote Sens., 10, 303.

Copernicus Emergency Management Service. https://emergency.copernicus.eu/ (14 July 2019).

Hess, L. L., Melack, J. M., Filoso, S., Wang, Y. 1995: Delineation of Inundated Area and Vegetation along the Amazon Floodplain with the SIR-C Synthetic Aperture Radar. IEEE Transactions on Geoscience and Remote Sensing, 33, 4, 896904. doi.org/10.1109/36.406675

Kaplan, G., Avdan, U., 2017: Object-based water body extraction model using Sentinel-2 satellite imagery. European Journal of Remote Sensing, 50, 1, 137-143. doi.org/10.1080/22797254.2017.1297540.

Martinis, S., Kersten, J., Twele, A., 2015: A fully automated TerraSAR-X based flood service. ISPRS Journal of Photogrammetry and Remote Sensing, Vol.104, 203-212. doi.org/10.1016/j.isprsjprs.2014.07.014.

McFeeters, S.K., 1996: The use of the Normalized Difference Water Index (NDWI) in the delineation of open water features. Int. J. Remote Sens. 17, 1425-1432. doi.org/10.1080/01431169608948714.

Stehman, S.V., 1997: Selecting and interpreting measures of thematic classification accuracy. Remote sensing of Environment, 62, 1, 77-89. doi.org/10.1016/S00344257(97)00083-7

Tsyganskaya, V., Martinis, S., Marzahn, P., Ludwig, R., 2018: Detection of Temporary Flooded Vegetation Using Sentinel-1 Time Series Data. Remote Sens. 10, 1286. doi.org/10.3390/rs10081286.

Twele, A., Cao, W., Plank, S., Martinis, S., 2016: Sentinel-1based flood mapping: a fully automated processing chain. International Journal of Remote Sensing, 37, 13, 2990-3004. doi.org/10.1080/01431161.2016.1192304. 